BNL-77298-2006-CP

\title{
Design of a Large-Area Fast Neutron Directional Detector
}

\author{
Peter E. Vanier ${ }^{1}$ Member IEEE, Leon Forman ${ }^{2}$ Cynthia Salwen ${ }^{1}$ \\ and Istvan Dioszegi ${ }^{I}$
}

${ }^{1}$ Brookhaven National Laboratory, Upton, NY 11973

${ }^{2}$ Ion Focus Technology, Miller Place, NY 11764

\author{
Nonproliferation and National Security Department \\ Detector Development and Testing Division \\ Brookhaven National Laboratory \\ P.O. Box 5000 \\ Upton, NY 11973-5000 \\ www.bnl.gov
}

\begin{abstract}
Notice: This manuscript has been authored by employees of Brookhaven Science Associates, LLC under Contract No. DE-AC02-98CH10886 with the U.S. Department of Energy. The publisher by accepting the manuscript for publication acknowledges that the United States Government retains a non-exclusive, paid-up, irrevocable, worldwide license to publish or reproduce the published form of this manuscript, or allow others to do so, for United States Government purposes.
\end{abstract}




\section{DISCLAIMER}

This report was prepared as an account of work sponsored by an agency of the United States Government. Neither the United States Government nor any agency thereof, nor any of their employees, nor any of their contractors, subcontractors, or their employees, makes any warranty, express or implied, or assumes any legal liability or responsibility for the accuracy, completeness, or any third party's use or the results of such use of any information, apparatus, product, or process disclosed, or represents that its use would not infringe privately owned rights. Reference herein to any specific commercial product, process, or service by trade name, trademark, manufacturer, or otherwise, does not necessarily constitute or imply its endorsement, recommendation, or favoring by the United States Government or any agency thereof or its contractors or subcontractors. The views and opinions of authors expressed herein do not necessarily state or reflect those of the United States Government or any agency thereof. 


\title{
Design of a Large-Area Fast Neutron Directional Detector
}

\author{
Peter E. Vanier, Member IEEE, Leon Forman, Cynthia Salwen and Istvan Dioszegi
}

\begin{abstract}
A large-area fast-neutron double-scatter directional detector and spectrometer is being constructed using 1-meterlong plastic scintillator paddles with photomultiplier tubes at both ends. The scintillators detect fast neutrons by proton recoil and also gamma rays by Compton scattering. The paddles are arranged in two parallel planes so that neutrons can be distinguished from muons and gamma rays by time of flight between the planes. The signal pulses are digitized with a time resolution of one gigasample per second. The location of an event along each paddle can be determined from the relative amplitudes or timing of the signals at the ends. The angle of deflection of a neutron in the first plane can be estimated from the energy deposited by the recoil proton, combined with the scattered neutron time-of-flight energy. Each scattering angle can be back-projected as a cone, and many intersecting cones define the incident neutron direction from a distant point source. Moreover, the total energy of each neutron can be obtained, allowing some regions of a fission source spectrum to be distinguished from background generated by cosmic rays. Monte Carlo calculations will be compared with measurements.
\end{abstract}

\section{INTRODUCTION}

$\mathrm{W}$ E have previously reported $[1,2]$ on a directional detector for fast neutrons based on double proton recoil in two planes of four $12.7-\mathrm{cm}$-diameter disk-shaped plastic scintillators equipped with photomultiplier tubes (PMTs). Those experiments grew out of earlier neutron time-of-flight measurements of the energy spectra of ${ }^{252} \mathrm{Cf}$ spontaneous fission and cosmic ray background [3]. In this paper, we show more detailed data obtained with the 8-disk system, and report on the design of the next generation detector, based on the same principles. One limitation of the first prototype is a low double-scatter event rate, especially for stand-off distances greater than 1 meter. Another drawback of the initial design is the limited number of scattering angles that can be intercepted by four detectors in the back plane. The new design uses large area scintillator paddles, which should provide increased absolute sensitivity and continuous horizontal positionsensing. The resulting images should be less streaky than those generated by the existing system.

As early as 1986, a double-scatter fast neutron detector with a long flight path was used to measure the energy spectrum of

Manuscript received November 28, 2006.

P. E. Vanier, Cynthia Salwen and Istvan Dioszegi are with the Nonproliferation and National Security Department, Brookhaven National Laboratory, Upton, NY 11973, USA (telephone: 631-344-3535, e-mail: vanier@bnl.gov).

Leon Forman is with Ion Focus Technology, Miller Place, NY, USA and is a consultant to Brookhaven National Laboratory. neutrons and the angular extent of a thermonuclear plasma source [4]. The objective of the present application of this technique is to locate a source of fission neutrons at long stand-off distances and distinguish such a source from the natural background produced by cosmic ray interactions with the atmosphere and the surroundings. The requirements for this task differ markedly from those needed in spacecraft that sense high-energy neutrons from the sun $[5,6]$. It is important to collect as many neutrons as possible in a short time, requiring large area, high sensitivity detectors. The neutron background fluxes at sea level are quite low, but gamma backgrounds are orders of magnitude greater, and must be rejected by the system in order to detect a weak neutron source. High precision angular resolution is not a critical parameter, provided that the data can indicate the approximate direction to the source. On the other hand the very massive systems constructed for basic nuclear physics are not optimized for the neutron energy range of $1-5 \mathrm{MeV}$, and are not easily transportable [7].

\section{PRINCIPLES OF OPERATION}

The geometry and principles of operation of the 8-element fast neutron double proton recoil directional detector have been previously described [1]. A schematic of the interleaved PMTs is shown in Figure 1. The energy of the recoiling proton in a front detector is measured by means of the peak area of the pulse recorded by the PMT, calibrated using the Compton edge for a 57Co source. The calibration process includes a conversion from electron energy to proton energy based on the equation recommended by the PMT manufacturer. The energy of the scattered neutron is determined by the time of flight from one front PMT to one back PMT. For the data reported in this paper, the angle subtended by each front detector at the back plane is about 16 degrees when the planes are $50 \mathrm{~cm}$ apart. The pulses are recorded by an Acqiris digitizer with $1 \mathrm{GHz}$ bandwidth, using one data point per nanosecond, and interpolating the leading edge half-way point to $0.1 \mathrm{~ns}$. That system has now been more carefully calibrated and exercised using a 252Cf source emitting 80,000 neutrons per second. The angle of scatter is related to the measured energies by the equations:

$$
\begin{aligned}
& \tan ^{2} \phi=E_{p} / E_{n s} \\
& E_{n}=E_{p}+E_{n s}
\end{aligned}
$$

where $\square$ is the scattering angle, Ep is the energy of the first recoil proton, Ens is the energy of the scattered neutron and En is the incident neutron energy. 


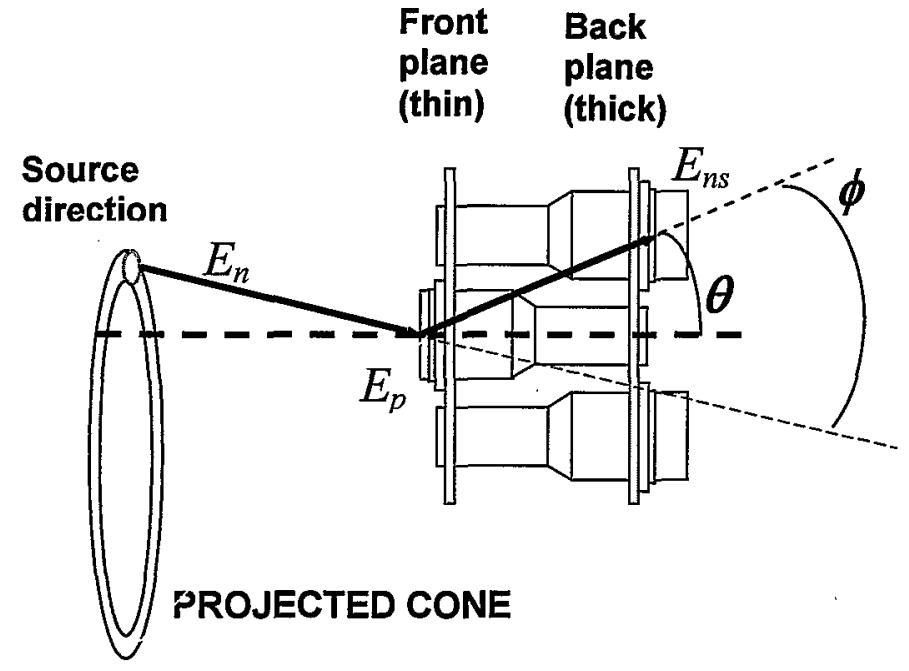

Fig. 1. Schematic of double scatter spectrometer.

A NIM logic system triggers the data acquisition for all events in which one front detector and one back detector exhibit a pulse with peak amplitude greater than the discriminator level of $0.25 \mathrm{~V}$, with a coincidence AND gate of width $1 \mathrm{~ms}$. This condition includes a number of types of events which must be sorted out by post-processing before an image can be obtained. Table 1 lists several of these types of coincidences, and comments about ways to avoid them.

TABLE 1. TYPES OF COINCIDENCE EVENTS

\begin{tabular}{|l|l|l|}
\hline \multicolumn{1}{|c|}{ Front detector } & \multicolumn{1}{|c|}{ Back detector } & Comment \\
\hline Single proton recoil & Proton recoils & Good event \\
\hline Multiple recoil & Proton recoils & $\begin{array}{l}\text { Bad event- use thin } \\
\text { front detector }\end{array}$ \\
\hline Gamma from source & Gamma from source & Short TOF \\
\hline Gamma from source & Proton recoil & Long TOF \\
\hline Proton recoil & Gamma from source & Negative "TOF" \\
\hline Background gamma & Background gamma & Random direction \\
\hline Background muon & Background muon & $\begin{array}{l}\text { Short TOF, large } \\
\text { pulses }\end{array}$ \\
\hline
\end{tabular}

If we had used liquid organic scintillators, it would have been possible to discriminate between gamma events and neutron events by means of the longer pulses produced by recoil protons. However, since most of the gamma coincidences were Compton scattering events, we were able to eliminate them simply by their short time of flight (less than 2 ns). We could also eliminate the high energy cosmic ray muons by selecting longer time of flight ( $>15 \mathrm{~ns}$ ) and by setting a maximum peak area corresponding to a proton energy of about $5 \mathrm{MeV}$. The muon events gave a peak in the energy spectrum corresponding to an energy greater than 10 $\mathrm{MeV}$. Accidental coincidences caused by uncorrelated gammas or neutrons were not considered a problem at these low background levels. However, one might have to consider their effect in a high-radiation active interrogation environment.
To illustrate some of the cuts made on the raw data, Figure 2 shows histograms of the time delays between pulses in the first and second detector planes for a typical run.

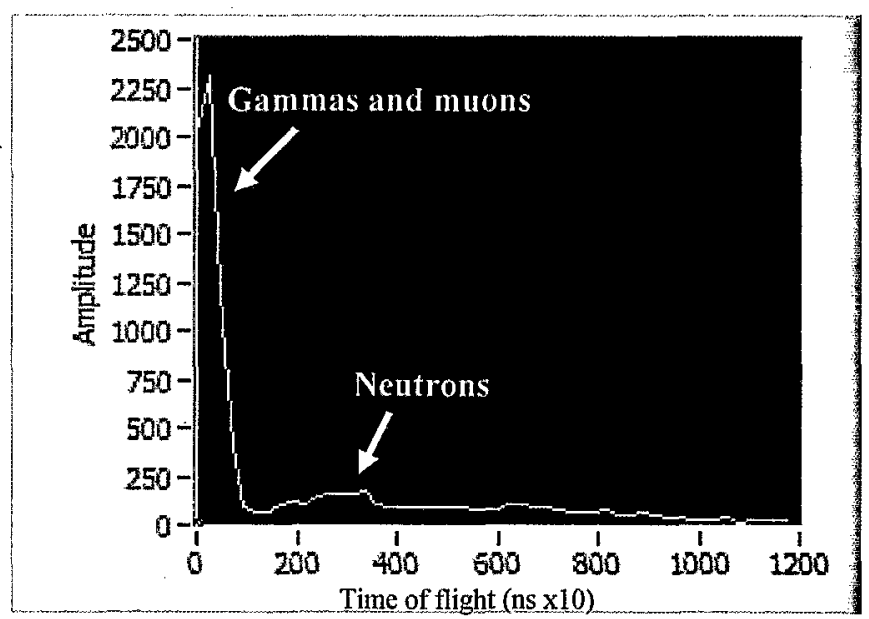

[a] complete coincidence data set

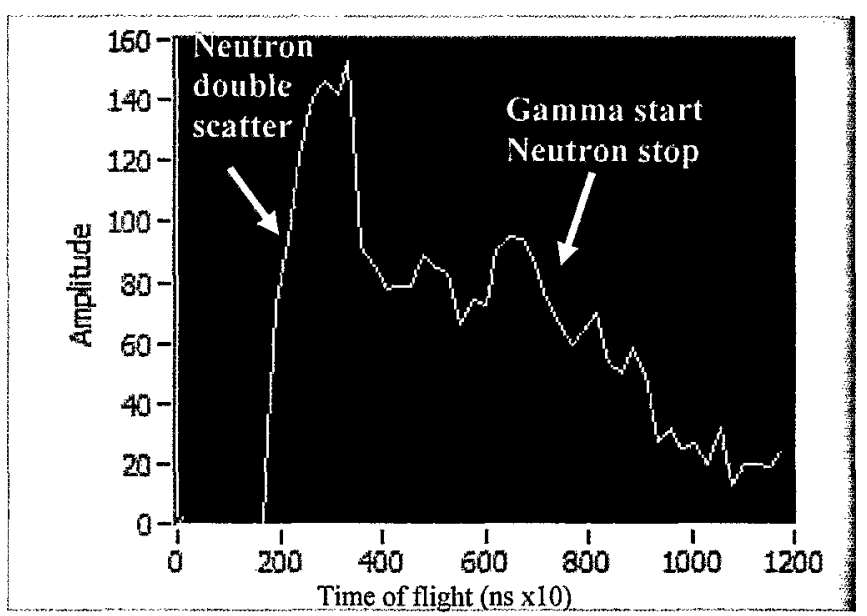

[b] events with time delays greater than $15 \mathrm{~ns}$

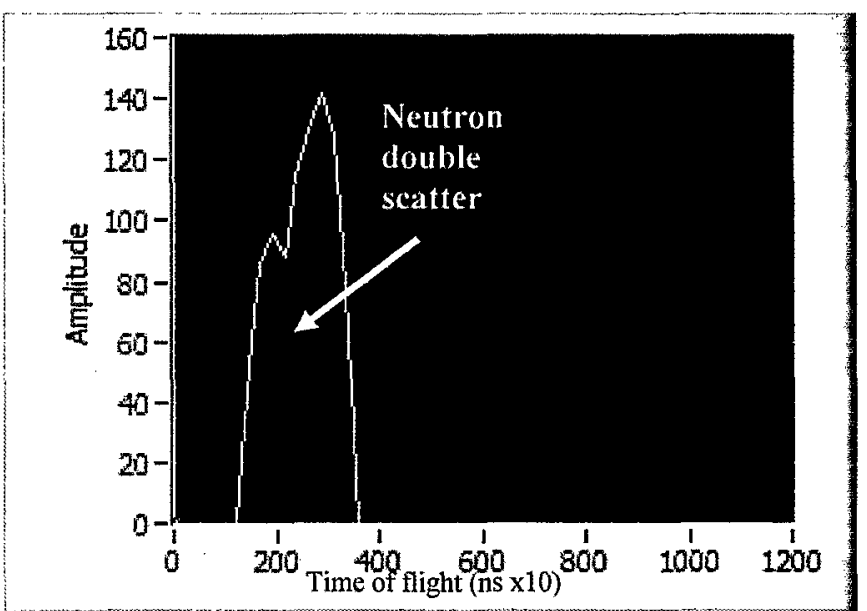

[c] time delays $>15 \mathrm{~ns}$ and $<35 \mathrm{~ns}$

Fig. 2. Discriminating types of coincidences by time of flight. 


\section{NEW DATA FROM 8-ELEMENT SYSTEM}

Figure 3 shows an example of an image obtained with the neutron source at a range of $2.9 \mathrm{~m}$, centered in the field of view. When displayed with an unadjusted grey scale, the overlapping ellipses show the 8-fold symmetry of the detector array. In the future, such artifacts :can be avoided by using position-sensitive detectors with uniform response. A horizontal profile of the data used in Figure 3 shows a full width at half maximum of $76 \mathrm{~cm}$, which corresponds to an angular resolution of 15 degrees, consistent with the angle subtended by each detector at the opposite plane.

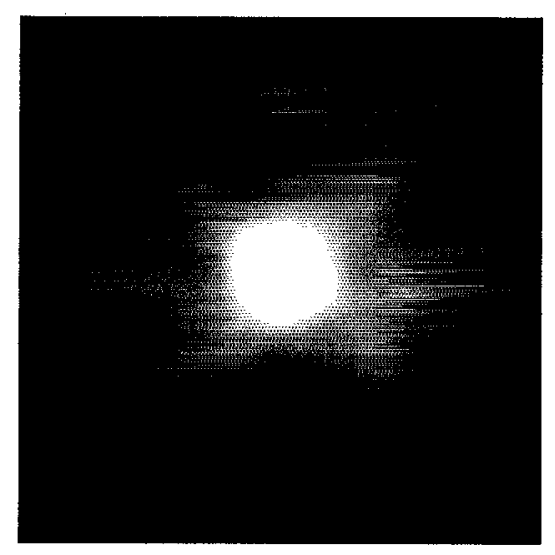

Fig. 3. Fast neutron image without contrast enhancement

By enhancing the contrast and setting a threshold, the brightest spot in the image can be emphasized, and the artifacts can be ignored. Figure 4 shows such an image acquired at a range of 9 meters with the source centered. This is the longest distance over which we have attempted to acquire an image with fast neutrons. If we were just counting neutrons without computing an image showing a localized source, it would be very difficult to distinguish the signal from natural background. The singles and coincidence count rates at this distance are essentially the same as measured with the source removed from the room.

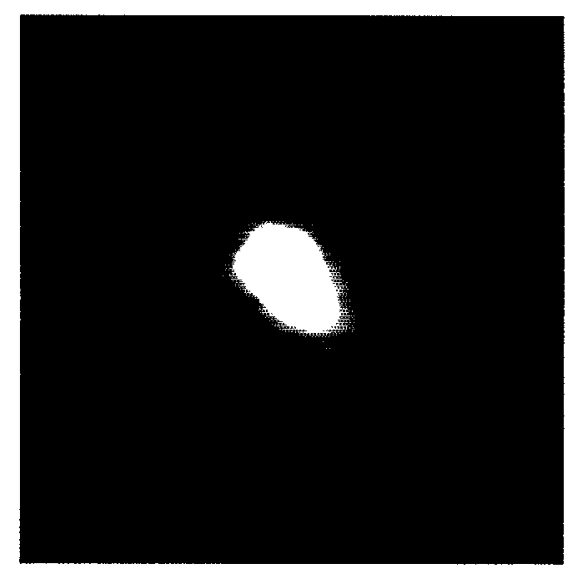

Fig. 4. Fast neutron image acquired at a range of 9 meters
Figure 5 shows a series of images produced by the 8element system with the source in different locations, at a range of $2.9 \mathrm{~m}$. The contrast of these images has been enhanced to remove traces of the non-overlapping parts of the ellipses. The field of view is approximately $5 \mathrm{~m} \mathrm{x} 5 \mathrm{~m}$, so the deflections of the brightest spots are in about the expected locations.

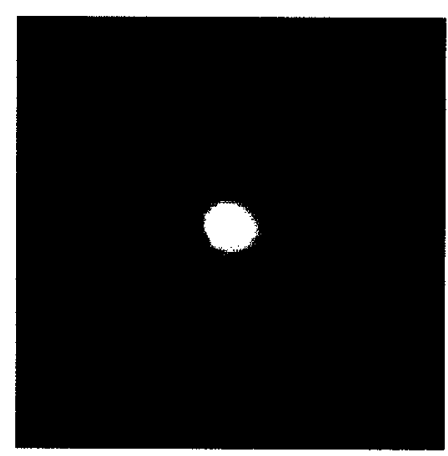

$[a] x=0 \mathrm{~cm}, y=0 \mathrm{~cm}$

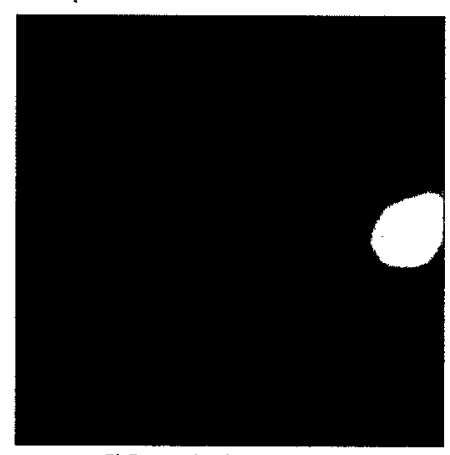

[b] $x=210 \mathrm{~cm}, y=0$

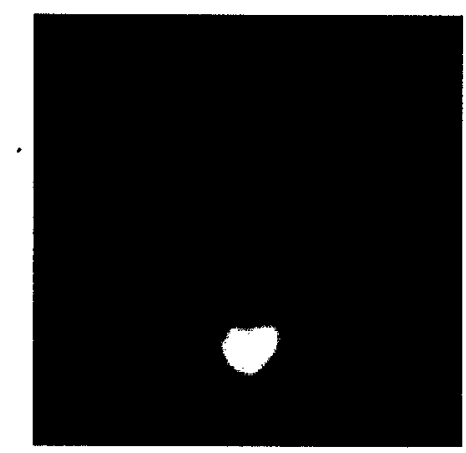

$[c] x=0, y=-116 \mathrm{~cm}$

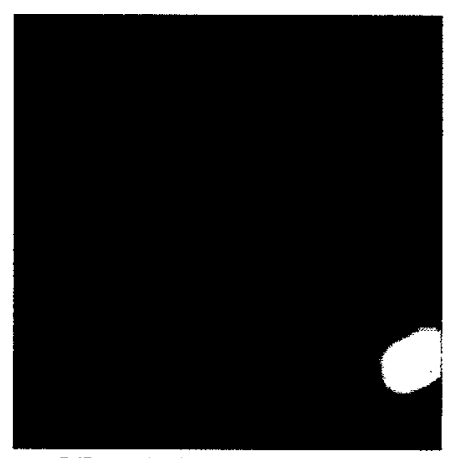

[d] $x=210 \mathrm{~cm}, y=-116 \mathrm{~cm}$

Fig. 5. Fast neutron images produced with 8-element system. 
Monte Carlo simulations of the 8-disk detector are in reasonable agreement with the experimental data. We are using the results of these calculations to understand the different types of scattering processes - in the detector and the environment - that contribute to the measured data. It should be possible to show by simulation how large a detector should be to obtain maximum performance, and what is the limitation on range imposed by scattering in the atmosphere.

\section{DESIGN OF LARGE AREA SYSTEM}

With the experience gained from operating the 8-disk system, we have designed the next generation large-area double-scatter directional fast-neutron detector incorporating a number of improvements. In the first place, the count rates should increase by about a factor of 8 because of the total area of the detector array, which is $100 \mathrm{~cm} \mathrm{x} 40 \mathrm{~cm}$. Second, the new design incorporates long scintillators paddles with continuous position sensing in the $\mathrm{x}$ direction, which should smooth out the images. Third, a new pulse processing system based on VME modules will be used to record the peak amplitudes and times of flight without the need to digitize the entire waveforms. This approach will allow higher count rates to be acquired without significant dead time, which will be more important as the total area of the detector is increased.

Figure 6 shows a photograph of the arrangement of two detector planes, each containing four scintillator paddles with PMTs at both ends. The spacing between the planes can be varied by means of a linear bearing on a track. Similar to the 8-element array, the front plane is $2 \mathrm{~cm}$ thick and the back plane is $5 \mathrm{~cm}$ thick. The planes are about $90 \%$ filled with scintillating material, compared to the fill factor of $27 \%$ for the interleaved disks.

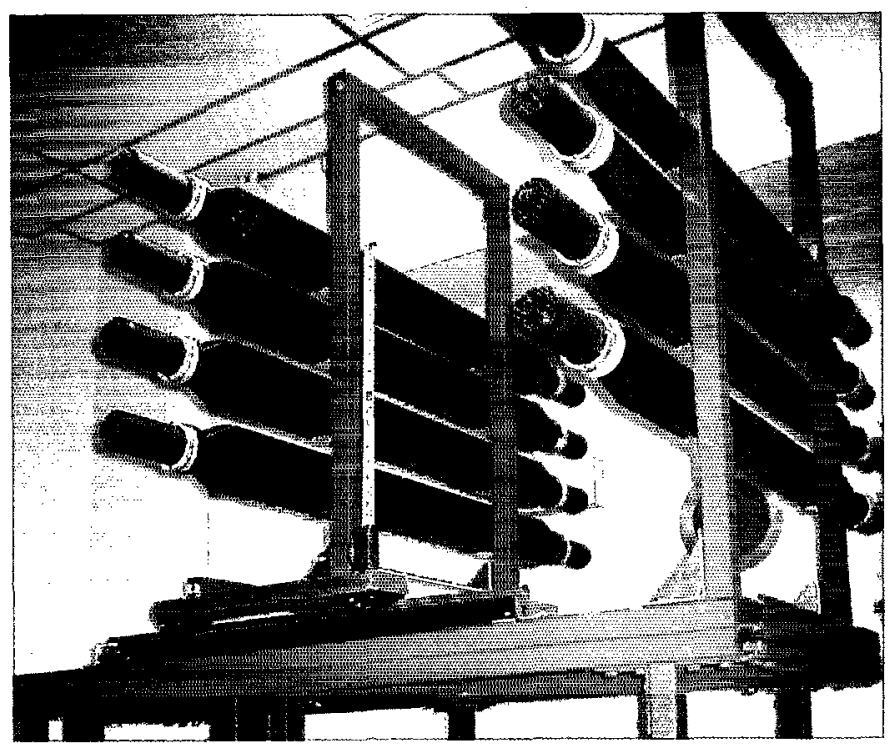

Fig. 6. New large area array of plastic scintillator paddles.

The time delay between pulses at the two ends of a paddle can be used as a measure of the location of the ionization event along the length of the paddle. We have begun the process of calibrating this position-sensitive response, as indicated in Figure 7. The data indicate that the position sensing is close to linear, and that it is possible to achieve the design goal of $10-\mathrm{cm}$ spatial resolution by means of pulse timing.

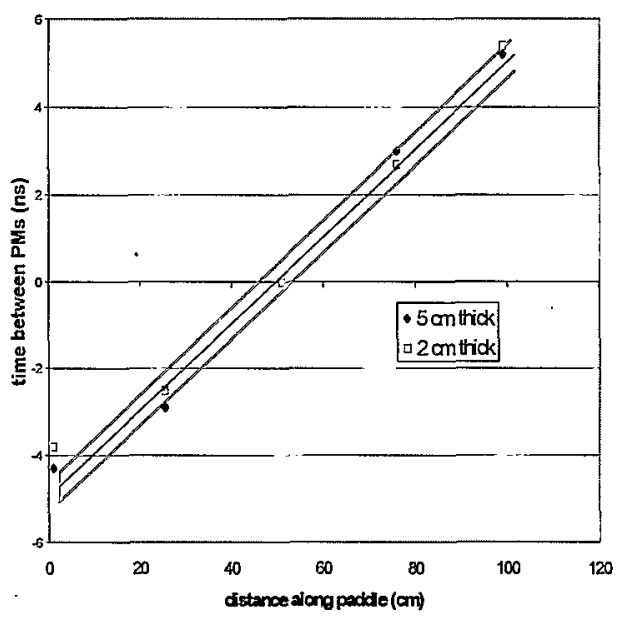

Fig. 7. Time delay between pulses in PMTs at both ends of a paddle as a function of source location.

\section{CONCLUSIONS}

We have demonstrated the principle of directional detection of a fission neutron source by double proton recoil at ranges of 1 to 9 meters using an array of 8 scintillating disks with a sensitive area of $500 \mathrm{~cm}^{2}$. We are now constructing a larger system with position-sensitive paddles that should reduce the time to acquire an image by about a factor of 8 .

\section{ACKNOWLEDGEMENTS}

This work was supported by U.S. Department of Energy, Office of Nonproliferation Research and Engineering, NA-22.

This manuscript has been authored by Brookhaven Science Associates, LLC under Contract No. DE-AC02-98CH1-886 with the U.S. Department of Energy. The United States Government retains, and the publisher, by accepting the article for publication, acknowledges, a world-wide license to publish or reproduce the published form of this manuscript, or allow others to do so, for the United States Government purposes.

\section{REFERENCES}

[1] Peter E. Vanier and Leon Forman, "An 8-element fast-neutron doublescatter directional detector", Proceedings of the SPIE - Hard X-ray and Gamma-ray Detector Physics VII, 5923-8, San Diego, CA, August, 2005.

[2] Peter E. Vanier and Leon Forman, "Demonstration of a Directional Fast Neutron Detector", Conference Record of IEEE Nuclear Science Symposium, Puerto Rico, 2005.

[3] Leon Forman, Peter E. Vanier and Keith E. Welsh, "Distinguishing spontaneous fission neutrons from cosmic-ray background", Proceedings of the SPIE - Conference 5541, Hard X-ray and Gamma-ray Detector Physics VI, Denver, CO, August, 2004. 
[4] S. E. Walker, A. M. Preszler, and W. A. Millard, "Double scatter neutron time-of-flight spectrometer as a plasma diagnostic", Rev. Sci. Instrum., 57, 1740-1742, Aug., 1986.

[5] R.S. Miller, J.R. Macri, M.L. McConnell, J.M. Ryan, E. Flückiger, and L. Desorgher, "SONTRAC: An imaging spectrometer for $\mathrm{MeV}$ neutrons," Nucl. Inst. Meth., vol. A505, pp. 36-40, 2003.

[6] Ulisse Bravar, Paul J. Bruillard, Erwin O. Flückiger, John R. Macri, Alec L. MacKinnon, Mark L. McConnell, Michael R. Moser, James M. Ryan, and Richard S. Woolf "Development of the Fast Neutron Imaging Telescope", 2005 IEEE Nuclear Science Symposium Conference Record, N6-3, 107, 2005.

[7] Modular Neutron Array (MONA) web page, Michigan State University, http://www.cord.edu/dept/physics/mona/. 